\title{
Facial Recognition Using Eigenfaces Approach
}

\author{
Mohd Noah A. Rahman, Armanadurni Abd Rahman, Afzaal H. Seyal and Nursuziana Kamarudin \\ Faculty of Business \& Computing \\ Institut Teknologi Brunei \\ Bandar Seri Begawan, Brunei Darussalam \\ noah.rahman@itb.edu.bn
}

\begin{abstract}
Face recognition has been researched extensively since the early 1950s and it is still an evolving domain for research. However, this application is relatively new in this country as compared to other biometric identifications. This paper seeks to find out the success rate of detection and recognition of the human faces using the eigenfaces method of the principal component analysis. It was conducted using the PCA algorithm on eigenfaces on 30 students using different images stored in a training database. From the experiment conducted, the PCA eigenfaces approach is able to deliver and produce high accuracy results.
\end{abstract}

Keywords-eigenfaces; face recognition; principal component analysis

\section{INTRODUCTION}

Facial recognition (FR) has been studied extensively since the early 50's. It has gain recognition since the emergence of severe attacks on buildings and public places on several occasions and the need of a FR is imminent. FR is defined as a biometric identification of a human's face and matching it against a library of known faces [1]. Therefore, this system does not require an individual to bring any identification in order to establish his or her identity. It uses images of a person's face for recognition and identification. This technology emerged in the middle of the twentieth century and was first introduced commercially in 1990s.

In this paper, a FR is basically a task of identifying an already detected face of a student as a known or unknown stored in a training database. The unknown face or test image will first go to the face detection in order to determine whether that image is a real 'face' or otherwise. Subsequently, FR will identify whether the detected face is someone known or unknown. It is by comparing the test image to a database of known faces stored in the database as shown in Figure I .

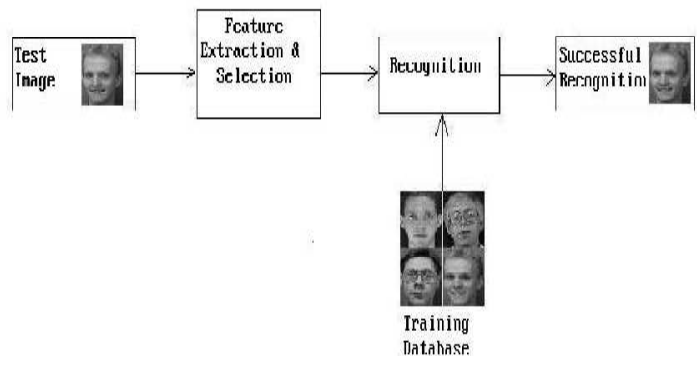

FIGURE I. FACE DETECTION AND FACE RECOGNITION [2].
The biometric method of identification has become an important mean of human identification. By comparing biometric identification to other biometric techniques like fingerprints, voice recognition and iris, FR is more efficient especially when it is being implemented in public places. Moreover, using FR does not require close interaction between the person and the identification system. Thus, this is a time efficient approach compares to other approaches such as fingerprints whereby the person needs to put his or her thumb on a slide.

FR techniques can be further classified as a geometric or a photo-metric. The geometric approach is a feature-based approach which looks at distinctive features, for instance the shape and position of the facial features. Photometric approach is an arithmetical approach that compute image into values therefore comparing them with the templates of training images in order to remove the variances for face identification. Some popular recognition algorithms include Principal Component Analysis (PCA) based eigenfaces, Hidden Markov Model (HMM) and the Linear Discriminate Analysis (LDA) among others.

\section{RELATED STUDIES}

FR has gained a lot of interest from researchers and it has become one of the most popular areas of research in computer vision and biometrics surveyed [3]. PCA was introduced in 1901 [4] and later in 1965 was proposed for pattern recognition [5]. After a span of thirty four years, Freeman and Tenenbaum proposed a bilinear model with a general framework [6]. It has also been widely considered as a successful application of image processing. Other than FR, there are multiple methods of biometric identification, for example, fingerprints and iris scans as illustrated in Table I [7]. 
TABLE I. FACE RECOGNITION APPLICATIONS (MARQUES, 2010)

\begin{tabular}{|c|c|}
\hline Domain & Application \\
\hline Biometric & $\begin{array}{ll}- & \text { Person identification } \\
- & \text { Automated identity } \\
\text { (border control) }\end{array}$ \\
\hline Information Security & $\begin{array}{ll}\text { - } & \text { Access security } \\
\text { - } & \text { Data privacy }\end{array}$ \\
\hline Access Management & $\begin{array}{ll}\text { - } & \text { Access authentication } \\
\text { - } & \text { Audit trails } \\
\text { - } & \text { Permission grant }\end{array}$ \\
\hline Law Enforcement & $\begin{array}{ll}\text { - } & \text { Video surveillance } \\
\text { - } & \text { Suspect identify } \\
\text { - } & \text { Simulated aging }\end{array}$ \\
\hline Personal Security & $\begin{array}{ll}- & \text { Home surveillance } \\
\text { - } & \text { Expression detection }\end{array}$ \\
\hline Entertainment and Leisure & $\begin{array}{ll} & \text { Video game } \\
\text { - } & \text { Photo camera }\end{array}$ \\
\hline
\end{tabular}

It is widely believed that FR is easier to use and secure as opposed to other forms of identification. As such, fingerprint has some limitations, as it is more difficult to retrieve due to fits orientation .

Thorat [8] has identified another weakness in FR which includes many systems are less effective if there are significant differences in facial expressions. Other conditions where FR does not work well include poor lighting, sunglasses, long hair or partially covered face, and low quality image acquisition.

Regardless of the problems and limitations, many researches on FR are still being carried out such as computer vision, optics, pattern recognition, neural networks, machine learning, psychology and so forth. In fact, more techniques are being invented each year such as 3D FR or recognition from video. To overcome such issues mentioned earlier, a Principal Factor Scrutiny method was proposed as an efficient method for face recognition [9].

A facial recognition device enables to view an image or a video of a person and later compares with one in the database's gallery by extracting features from an image of the person's face. Face detection is the first stage in the recognition process where all faces are distinguished from non-faces. It is easy for human being to recognize faces even with different appearances such as different hairstyle, with and without glasses, contact lenses among others.

FR is the second stage after face detection has been done. One way to do this is by feature extractions. There are two approaches for feature extraction; local and global. For local feature, it extracts eyes, nose and mouth information. The coordinates of a set of features from the photographs are extracted and then used by the computer for recognition. For global feature, it extracts features from the whole image that is known as the holistic method. Turk and Pentland [10] discovered the residual error could be used to detect images of faces using eigenfaces.

\section{RESEARCH APPROACH}

PCA is also known as eigenfaces having a feature extraction algorithm. Kirby and Sirovich [11] applied PCA for faces representation while Turk and Pentland [10] extended PCA for face recognition. Subsequently, Mittal and Walia [12] introduced a fast PCA based facial recognition algorithm.
Furthermore, Hossein et al. [13] presented FR using PCA, LDA and neural networks.

A set of eigenfaces can be generated by performing a PCA on a large set of images that depict various human faces as shown in Figure II. There are two stages in eigenfaces which involve learning and recognition. The learning stage involves the collection of images. These images are called the training images or training set. The recognition stage will identify whose face it belongs to.

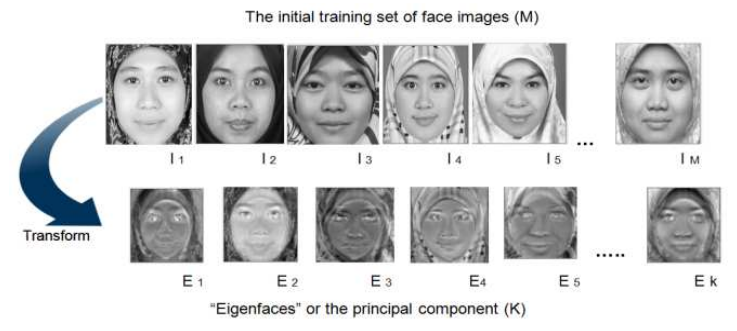

FIGURE II. TRANSFORMATION PROCESS

The PCA will transform the training faces of $\mathrm{M}$ into a smaller set of $\mathrm{K}$ eigenfaces which evaluates into $\mathrm{K}<\mathrm{M}$. Each image is denoted in terms of these eigenfaces with assigned weights. This transformation is defined wherein the first principal component or eigenface which always presents the most important features of the training set and each consecutive component would display the next dominant feature. As an example, assuming the training set consists of 200 face images. Therefore $\mathrm{M}$ will be 200. PCA will transform $\mathrm{M}$ into a small set of $\mathrm{K}$ by removing eigenfaces that contain awful data.

PCA will only select the first 50 eigenfaces which carry useful information and discard the rest of the eigenfaces that are not important to the images that carry more noises. To recognize the test image, the difference between it and each training image must be calculated. Each image is actually made up of proportions of all $\mathrm{K}$ "features". It is also possible to rebuild each face image from the training set, by combining the eigenfaces.

This particular project is to experiment the face recognition for student verification for a library system. Therefore, the training set is a set of students' images. An individual image has been taken to be recognized, i.e. a passport size photograph of each student. In addition, each face image that is collected for the training set contains some information about the student such as faceID, faceName, rollNumber, course and department.

\section{EXPERIMENTAL RESULT AND ANALYSIS}

The computer used in this experiment is a PC with $32 \mathrm{~GB}$ RAM, a webcam and a terabyte of hard disk space utilising MS Access DBMS which was proven to be beneficial in storing data [14]. The programming languages used are C, C\# and C++ which provide excellent programming skills [15]. 


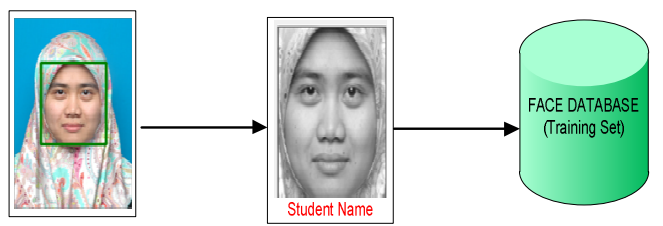

FIGURE III. TRANSFORMATION PROCESS

The database as shown in FigureIII contains a collection of faces of students (training set) where the system is built to recognize. The training database contains 46 images of 30 individual students, consisting of students from the computing programmes of the university. For testing, 35 images of 30 known and 5 unknown students were used. To test the recognition performances in real environments, test image of the student are taken using the webcam.

From the test conducted, 21 face images were successfully recognized, while 3 faces were not detected, and 6 faces were able to be detected but not recognized by the system. In the original and testing images, it can be seen that the students have different postures and facial expressions therefore the system failed to successfully match both images

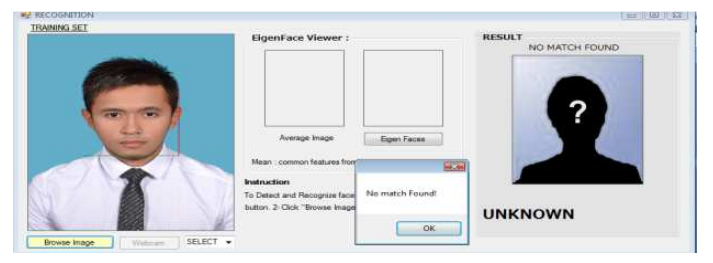

FIGURE IV. IMAGE OF UNKNOWN FACES AND UNMATCHED

In a case where an unknown image is detected, the system will not be able to recognise the fixtures that are stored in the database as shown in Figure IV above.

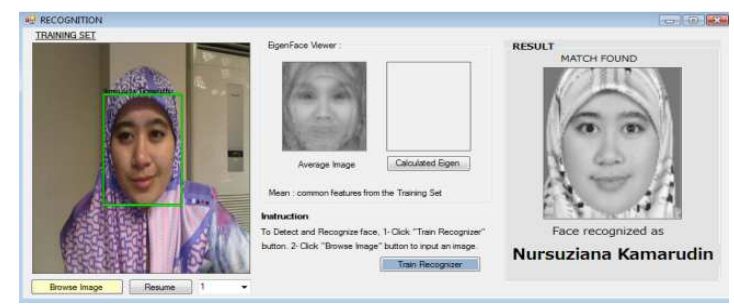

FIGURE V. IMAGE FROM A WEBCAM

The system is able to match and recognize the image taken from a webcam with the images stored in the training database as depicted in Figure V.

\section{CONCLUSION}

Overall, the system has managed to meet the aims and objectives. It works not only on static images but also on dynamic images including capturing face using webcam. In addition, it can recognize multiple face images of the same student and able to measure the successful rate of FR.
From the experiments conducted, the accuracy rate of facial recognition from a single image is very high. This proves that even though this technique has existed over the years, it could still work relatively well especially in a controlled environment such as images with the same lighting, position, and expression.

Finally, it can be deduced that the accuracy of face recognition is up to an average of $70 \%$ accuracy. It is vital to include the full frontal face with less facial expression in order to ensure the successful rate of the PCA algorithm.

Future studies for this application can be extended on an on-line and real time resided in one of the university's application servers.

\section{REFERENCES}

[1] J.Shermina, "Face recognition system using multilinear PCA and locality preserving projection," Proc. 2011 IEEE GCC Conference and Exhibition, pp. 283-286, 2011.

[2] S. Bag and G. Sanyal, "An efficient face recognition approach using PCA and mnimum distance classifier," Proc. 2011 International Conference on Image Processing (ICIIP), 2011.

[3] W. Chao, R. Chellapa, P.J Phillips and A. Rosenfeld, "Face recognition: A literature survey," ACM Computing Surveys, vol. 35(4), pp. 399-458, 2003.

[4] K. Pearson, "On lines and planes of closed fit to ssystems of points in space," Philosophical Magazine, vol. 2(6), pp. 559-572, 1901.

[5] S. Watanabe, "Karhunen-Loeve expansion and factor analysis theoretical remarks and applications," Proc. Of the 4th Prague conference on Information Theory, 1965.

[6] W.T. Freeman and J.B. Tenenbaum, "Learning bilinear models for twofactor problems in vision," Proc. Of the IEEE Computer Society Conference on Computer Vision and Pattern Recognition,”, pp. 554-560, 1997.

[7] I. Marques, "Face Recognition Algorithms," http://www.ehu.es/ccwintco/uploads/e/eb/PFC-IonMarques.pdf, 2010

[8] S.B. Thorat, "Facial recognition technology: An analysis with scope in India," Journal of Computer Science and Information Security, vol. 8(1), 2010.

[9] C.V. Arulkumar, G. Selvayinayagam and J. Vasuki, "Enhancement in face recognition using PFS using Matlab," International Journal of Computer Science \& Management Research, vol. 1(1), pp. 282-288, 2012.

[10] M. Turk and A. Pentland, "'"Eigenfaces for recognition," Journal of Cognitive Neuroscience, vol. 3(1), pp. 71-86, 1991.

[11] M. Kirby and L. Sirovich, "Application of Karhunen-Loeve procedure for the characterisation of human faces," IEEE Transactions on Pattern Analysis and Machine Intelligence, vol. 12, pp. 103-108, 1990.

[12] N. Mittal and F. Walia, "Face recognition using improved fast PCA," IEEE Congress on Image and Signal Processing, vol. 1, pp.554-558, 2008.

[13] A.S. Hossein, B.Z. Heidari and C.H. Dehghani, "A new face recognition using PCA, LDA and neural network, " International Journal of Computer Science and Engineering, vol. 2(4), 2008

[14] M.N. Rahman, Y. Mohamed, A.H. Seyal and I. Mashud, "The significance of database training and perceived benefits on the use of DBMS," Seameo Voctech Journal, vol. 9(1), pp. 42-53, 2009.

[15] M.N. Rahman, M.M. Rahim and A.H. Seyal, "Requisite skills of entrylevel programmers: An empirical study in Brunei Darussalam," Journal of Educational Computing Research, vol. 21(3), pp. 305-323, 1999. 